\title{
Caractérisation chimique des grès khmers du Buriram (Thaillande)
}

Valérie Thirion-Merle, Armand Desbat et Ranet Hong

\section{(2) OpenEdition}

Journals

Édition électronique

URL : https://journals.openedition.org/baefe/4504

DOI : $10.4000 /$ baefe. 4504

ISSN : 2732-687X

Éditeur

ResEFE

Référence électronique

Armand Desbat, Valérie Thirion-Merle et Ranet Hong, « Caractérisation chimique des grès khmers du Buriram (Thaïlande) » [notice archéologique], Bulletin archéologique des Écoles françaises à l'étranger [En ligne], Asie du Sud-Est continentale, mis en ligne le 29 novembre 2021, consulté le 28 juin 2022. URL : http://journals.openedition.org/baefe/4504; DOI : https://doi.org/10.4000/baefe.4504

Ce document a été généré automatiquement le 1 décembre 2021.

\section{c) (i) $९$}

Le Bulletin archéologique des Écoles françaises à l'étranger est mise à disposition selon les termes de la Licence Creative Commons Attribution - Pas d'Utilisation Commerciale - Pas de Modification 4.0 International. 


\title{
Caractérisation chimique des grès khmers du Buriram (Thailande)
}

\author{
Valérie Thirion-Merle, Armand Desbat et Ranet Hong
}

\section{NOTE DE L'AUTEUR}

Partenariats institutionnels : CNRS

Établissement éditeur : EFEO

Établissements porteurs de l'opération : EFEO, CNRS

1 Les grès angkoriens constituent une production céramique originale, caractéristique de la période angkorienne (IXe-XIVe siècle a. D.). De nombreuses études ont été réalisées à partir de découvertes sur les sites de consommation mais aussi depuis la découverte des sites de production autour d'Angkor et dans d'autres provinces ${ }^{1}$. Mais ces recherches se limitaient bien souvent à quelques études de cas. Le programme Cerangkor ${ }^{2}$ s'est $^{\prime}$ distingué par son ampleur en s'attachant à définir, pour tous les sites d'ateliers connus, les caractéristiques de la production sur les plans typologique et technique mais également du point de vue des compositions géochimiques. Un vaste programme d'analyses chimiques a été entrepris de façon à définir les compositions chimiques de chaque catégorie de grès identifiée. Six groupes de fours sont connus aux alentours de la cité d'Angkor au Cambodge et ont fonctionné entre les IXe et xIVe siècle. ${ }^{3}$ Des groupes de références géochimiques ont été définis, permettant d'étudier la diffusion de ces ateliers d'une part et de préciser leur durée de vie par l'analyse des céramiques des sites de consommation d'autre part. ${ }^{4}$

2 À partir du xie siècle émergent d'autres centres producteurs notamment au Buriram aujourd'hui une des provinces de Thaillande. Pas moins d'une centaine de fours produisant différentes catégories de grès ont été identifiés. ${ }^{5}$ Malheureusement aujourd'hui la plus grande partie de ces fours identifiés dans les années 80 a disparu.

3 Entre 2015 et 2017, l'équipe de Cerangkor a mené plusieurs enquêtes sur les sites de fours encore visibles du Buriram (districts de Ban Kruat et Lahansai) ainsi qu'à Ban 
Sawai (province de Surin) et Ban Ya Kha (province de Nakhon Ratchasima) où d'autres sites ont été suspectés. ${ }^{6}$ Nos prospections se sont surtout concentrées sur les sites de fours encore visibles autour de la ville de Ban Kruat à partir des cartes établies par le Fine Art Department (1989) et surtout grâce à l'aide de Mr Surapol Tawankar, professeur au lycée de Ban Kruat qui connaît parfaitement les sites de fours et nous a servi de guide. Nos prospections ont également concerné plusieurs sites situés plus au sud dans le district du Lahan Saï (fig. 1). Sur chaque site de fours plusieurs catégories de grès ont pu être produites, des grès non glaçurés ou des grès glaçurés. Ces derniers peuvent être à glaçure brune, à glaçure verte ou bicolores; ce qui est une des caractéristiques des fours de cette province.

Fig. 1. Localisation des sites de fours autour de Ban Kruat et dans le district du Lahan Saï (Buriram, Thaïlande).

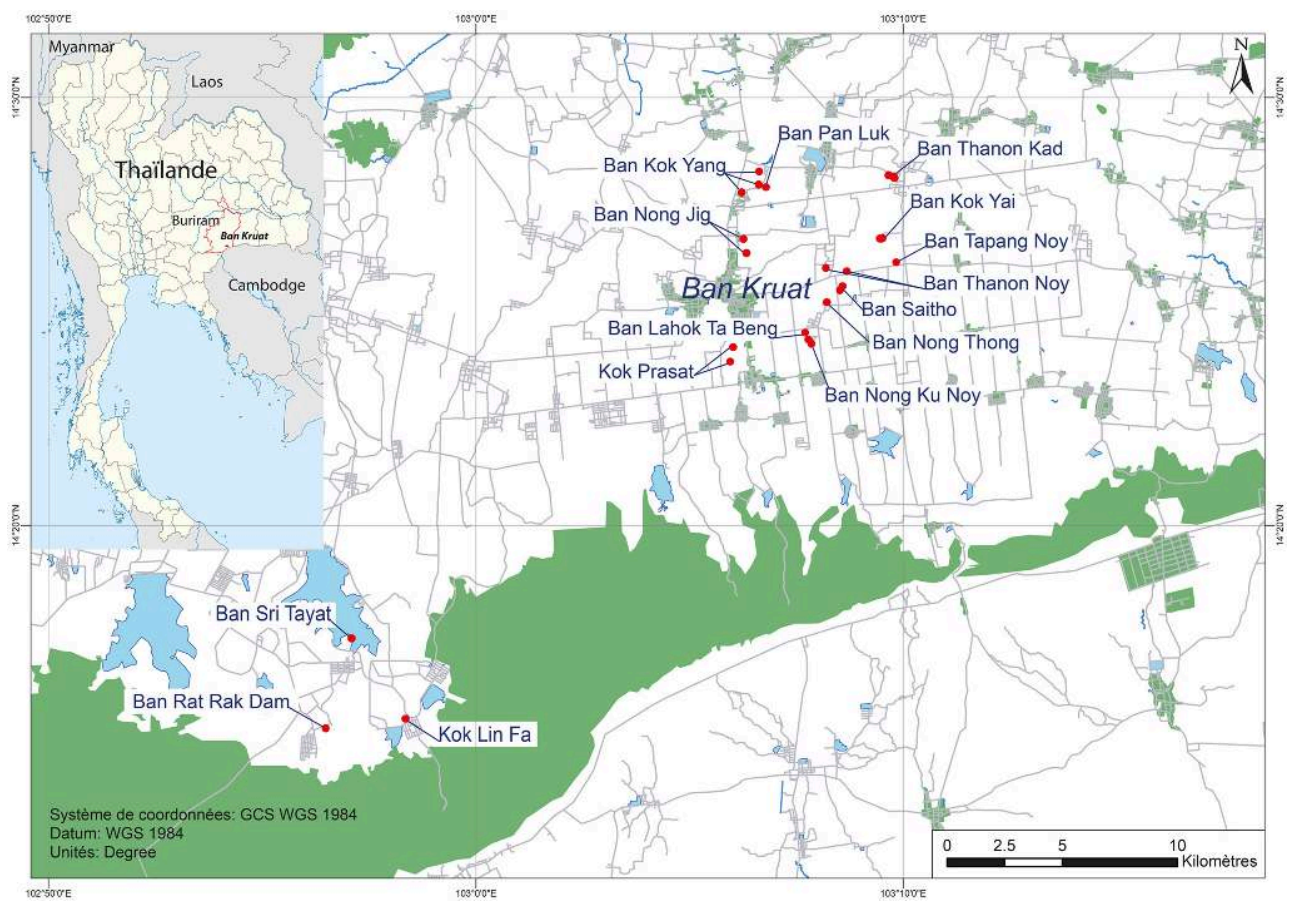

Fond de carte issu du Projet OpenStreetMap, depuis le serveur de téléchargement gratuit Geofabrik.

L'objectif de cet article est de présenter les résultats obtenus à partir des analyses chimiques des différents types de grès retrouvés sur les sites producteurs du Buriram. Nous disposons dorénavant d'environ 350 références pour caractériser les productions de grès à glaçure verte (fig. 2 et 3), grès à glaçure brune (fig. 4 à 6) et grès non glaçurés de plus d'une quinzaine de sites de fours dispersés autour de Ban Kruat et dans le district du Lahan Saï. Ces analyses nous ont permis d'établir des groupes de références relativement solides qui sont détaillés ici. 
Fig. 2. Planche de grès à glaçure verte retrouvés sur le site de Ban Rat Rak Dam.

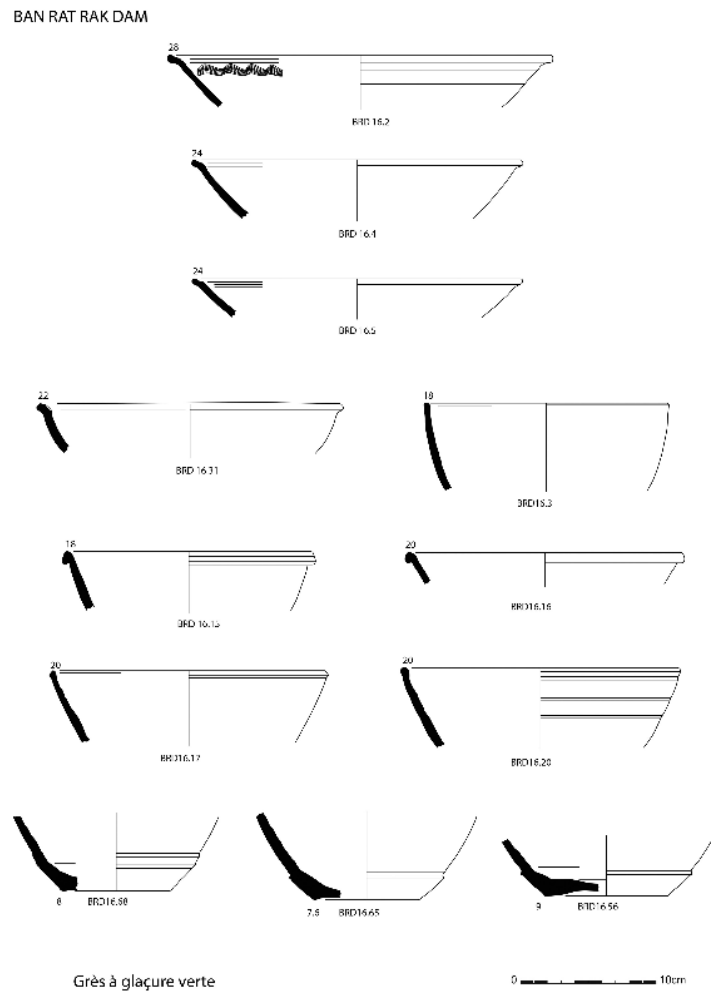

Fig. 3. Photo d'un bol à glaçure verte.

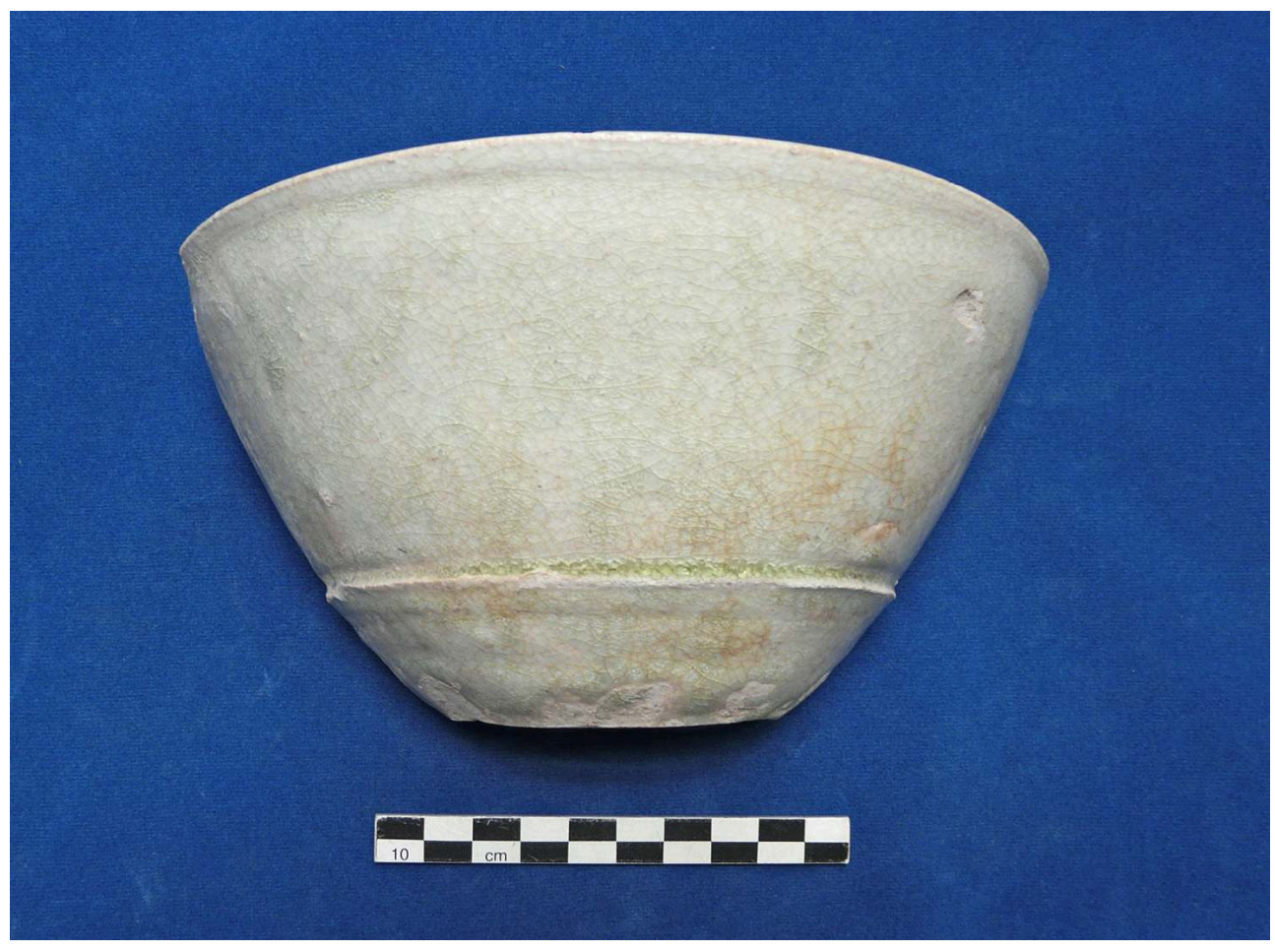

A. Desbat. 
Fig. 4. Planche de grès à glaçure brune retrouvés sur le site de Ban Rat Rak Dam.

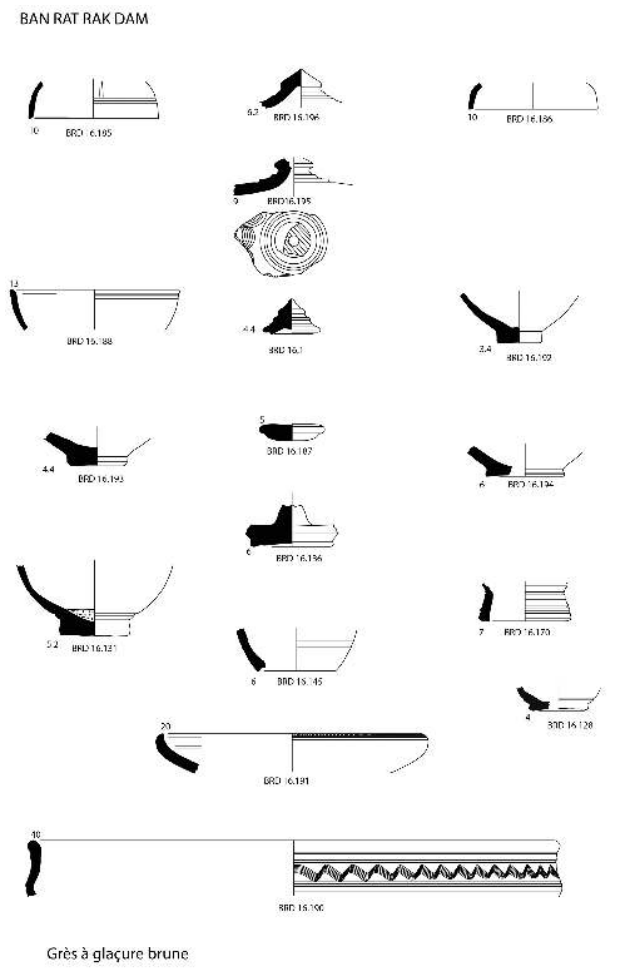

Fig. 5. Planche de grès à glaçure brun/noir retrouvés sur le site de Ban Kok Yai.

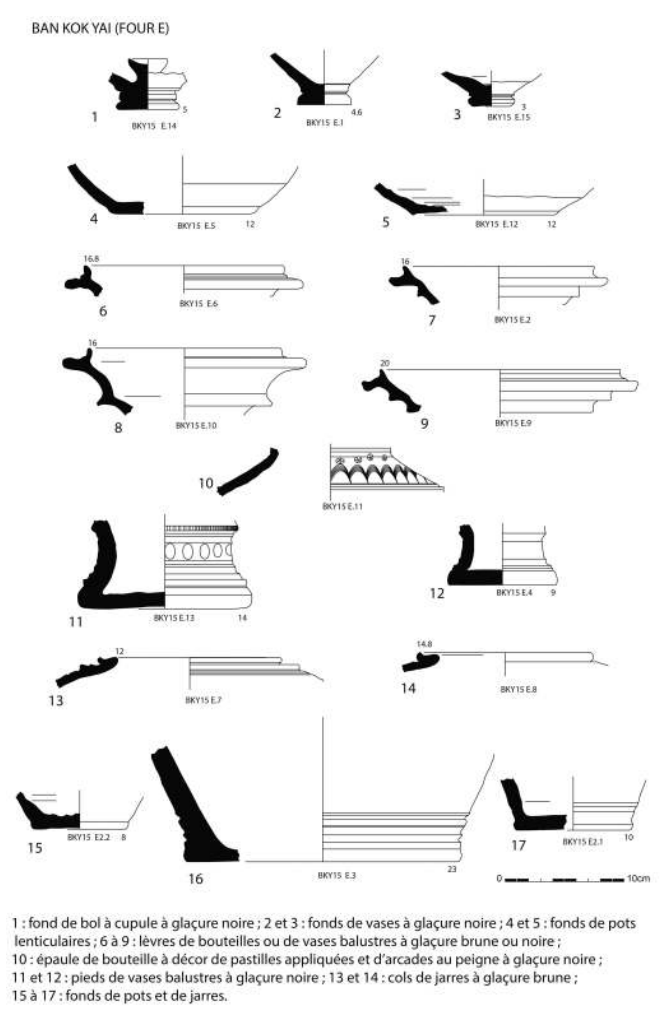


Fig. 6. Photo des tessons de grès à glaçure brun/noir retrouvés sur le site de Ban Kok Yai.

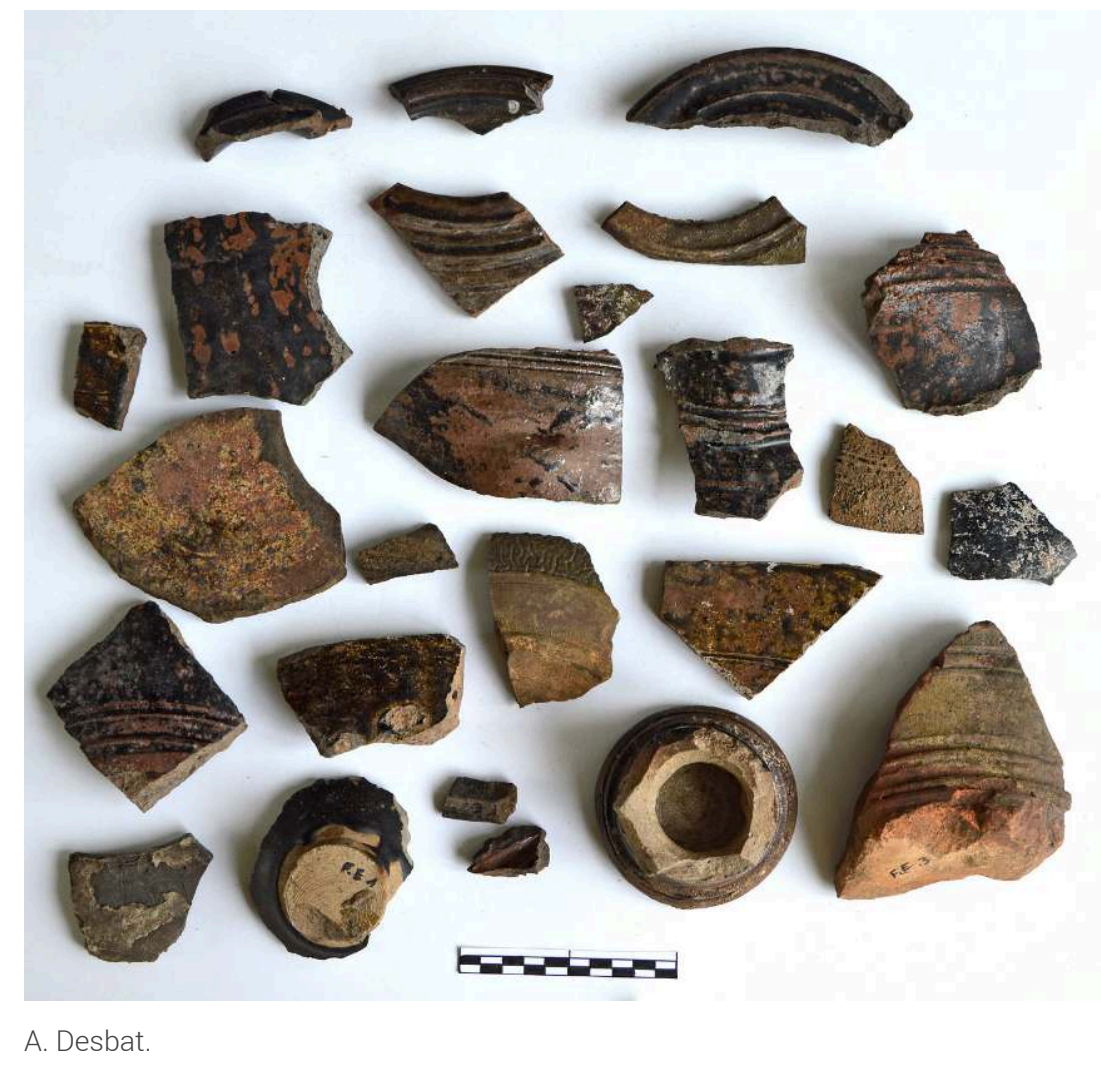

\section{Observations macroscopiques}

Des observations macroscopiques ont tout d'abord été réalisées sur les échantillons analysés. Ces observations s'intéressent à l'aspect général de la pâte céramique, à la matrice argileuse et aux inclusions présentes (forme, couleur, taille, abondance). Elles ont été réalisées à la loupe binoculaire sur cassure fraîche et/ou coupe et montrent que la pâte des grès à glaçure verte est essentiellement fine, de couleur claire (beige, grise voire blanche) et contient de très nombreux grains blancs et/ou translucides très fins mais très peu de nodules d'oxyde de fer, au contraire des grès non glaçurés et grès à glaçure brune qui présentent pour une grande partie d'entre eux des pâtes plus grossières, de teintes foncées et contenant de nombreux nodules d'oxydes de fer de tailles variées ainsi que de gros grains de quartz (fig. 7). On peut donc déduire de ces premières observations que les grès à glaçure verte ont été produits à partir de matières premières argileuses nettement différentes de celles utilisées pour les grès à glaçure brune ou les grès non glaçurés. Par contre, ces deux dernières catégories de grès semblent avoir été produites à partir de matières premières similaires.

6 Néanmoins sur seulement trois sites à proximité de Ban Kruat, à savoir les sites de Ban La Hok Ta Beng, Ban Nong Ku Noy et Ban Kok Yai, quelques grès à glaçure brune présentent exactement la même pâte que les grès à glaçure verte. A Ban Sri Tayat, district du Lahan Saï, un seul exemplaire de ce type a été découvert. Les analyses ont montré que leurs compositions étaient strictement similaires à celle des grès à glaçure verte. Les potiers ont donc parfois utilisé la même argile pauvre en oxyde de fer pour 
fabriquer des grès glaçurés verts ou bruns. Ces cas sont toutefois assez rares et pourraient correspondre aux productions les plus anciennes.

Fig. 7. Photographies des pâtes céramiques de quelques grès types (grossissement 1).

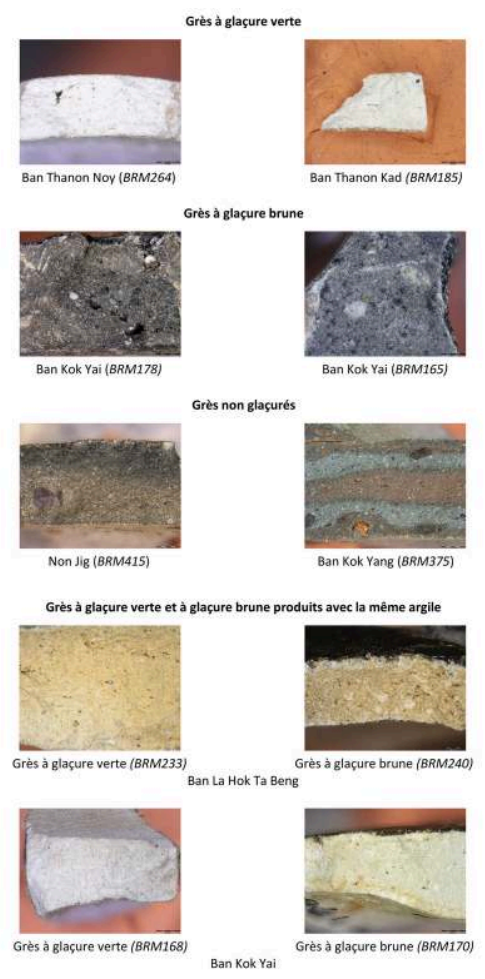

V. Thirion-Merle.

\section{Les analyses chimiques}

7 La composition chimique de chaque échantillon de grès a été déterminée par spectrométrie de fluorescence $\mathrm{X}$ à dispersion de longueur d'onde (WDS-XRF), au laboratoire d'archéologie et d'archéométrie de Lyon(France), à l'aide d'un spectromètre Bruker S8 Tiger doté d'une source d'excitation Rh. Cette technique nécessite de prélever et préparer les échantillons selon un protocole précis : un petit prélèvement est effectué sur chaque échantillon à l'aide d'une scie diamantée. Les surfaces extérieures, susceptibles d'être altérées chimiquement lors de l'enfouissement sont éliminées. Le grillage des prélèvements à $950^{\circ} \mathrm{C}$ (nécessaire pour éliminer l'eau, les substances volatiles et organiques) est suivi du broyage à l'aide d'un broyeur à galet en carbure de tungstène. Un total de $800 \mathrm{mg}$ du prélèvement en poudre est ensuite ajouté à $3200 \mathrm{mg}$ de fondant chimique (mélange de métaborate et tétraborate de lithium), le mélange obtenu est ensuite chauffé pour produire un disque de verre. La mesure peut alors être effectuée sur ce disque de verre de composition parfaitement homogène, qui correspond à la composition chimique moyenne représentative du matériau initial.

Du fait du procédé de préparation des échantillons mis en œuvre, cette technique fournit la composition chimique globale d'une céramique (matrice plus inclusions) et par conséquent du matériau utilisé pour sa fabrication. Pour chaque échantillon, 
24 composants sont déterminés sur la base de courbes d'étalonnage établies avec 40 géostandards internationaux ${ }^{7}$.

9 Les résultats de ces analyses chimiques ont bien confirmé que les grès non glaçurés et grès à glaçure brune présentaient des compositions similaires, nettement différentes de celles des grès à glaçure verte ${ }^{8}$. Cela peut très simplement se vérifier à partir des diagrammes binaires mettant en relation, d'une part, les teneurs en oxyde de fer et en oxyde de manganèse des grès non glaçurés (GNG), des grès à glaçure brune (GGB) et des grès à glaçure verte (GGV), et d'autre part les teneurs en silice et alumine pour ces mêmes grès (fig. 8). D'autres éléments chimiques participent à cette distinction indiquant ainsi que les matériaux argileux sont nettement différents. Les potiers ont sélectionné leurs argiles en fonction de ce qu'ils souhaitaient produire.

Fig. 8. Diagrammes binaires mettant en relation, d'une part, les teneurs en oxyde de fer et en oxyde de manganèse et les teneurs en silice et alumine, d'autre part, des grès non glaçurés (GNG), des grès à glaçure brune (GGB) et des grès à glaçure verte (GGV) des sites de fours du Buriram.
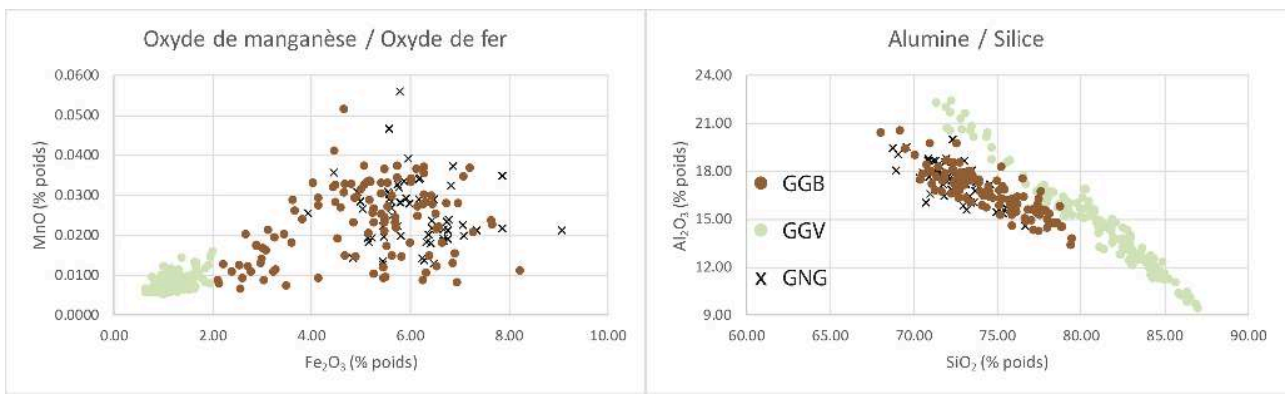

Toutefois, les analyses chimiques ont également confirmé que sur certains sites de fours, notamment Ban La Hok Ta Beng, Ban Saitho et Ban Kok Yai, les potiers ont utilisé exactement les mêmes argiles pour produire les grès à glaçure brune et ceux à glaçure verte, dans la mesure où l'on retrouve ces deux catégories de grès dans les mêmes groupes de composition.

11 À la lumière de ces résultats, les groupes de référence seront présentés pour chaque catégorie de grès.

\section{Les grès à glaçure verte}

La comparaison des compositions chimiques de l'ensemble des grès à glaçure verte analysés du Buriram (sites des districts de Ban Kruat et du Lahan Saï) a été réalisée à partir d'une classification ascendante hiérarchique ${ }^{9}$, méthode statistique qui permet de mettre en évidence des groupes de composition rassemblant des échantillons présentant de fortes affinités de compositions (fig. 9). 
Fig. 9. Classification ascendante hiérarchique des grès à glaçure verte retenus comme références du Buriram, réalisée sur 17 éléments chimiques, $(n=170)$.

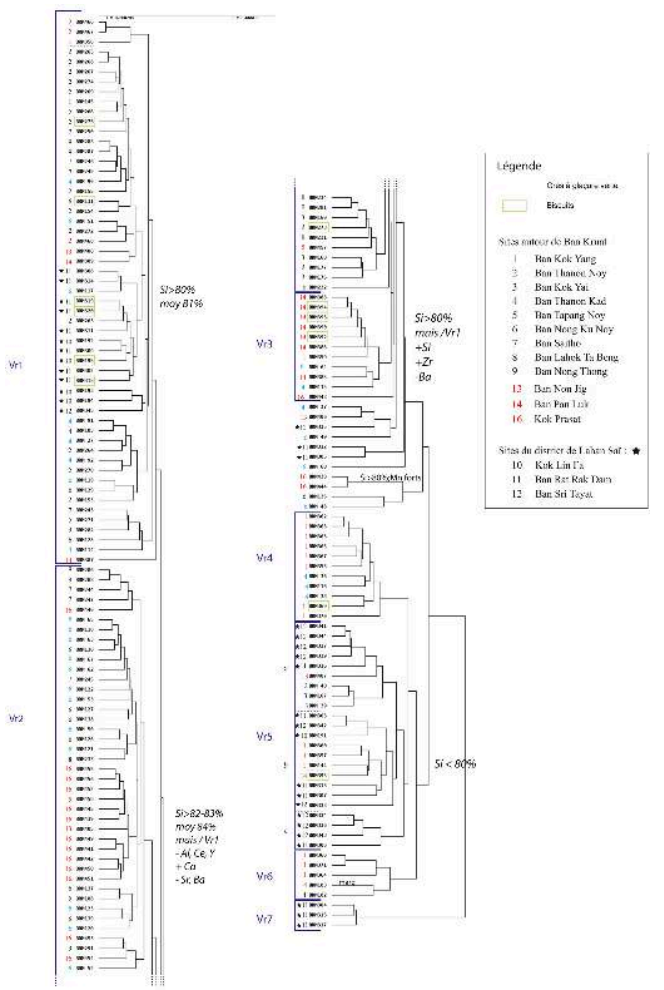

13 Cette classification fait apparaître un grand nombre de groupes de compositions dénommés Vr1 à Vr7, reflets de la diversité des matériaux argileux utilisés. Les compositions moyennes des différents groupes établis sont données dans le tableau 1.

La majeure partie des grès à glaçure verte ont été produits avec des matières premières ayant des teneurs en silice très élevées ( $>80 \%$, voire $83 \%)$ et des teneurs en la plupart des autres éléments chimiques faibles, et notamment les teneurs en alcalins et alcalinoterreux (grès des groupes Vr1 à Vr3 + marginaux internes). Ce qui dénote l'utilisation de matériaux argileux kaolinitiques (contenant une forte proportion de kaolinite, minéral argileux de formule chimique $\left.\mathrm{Al}_{2} \mathrm{Si}_{2} \mathrm{O}_{5}(\mathrm{OH})_{4}\right)$. Pour certains groupes les teneurs en alumine sont plus élevées suggérant une argile plus kaolinitique.

La corrélation inverse silice/alumine est vraisemblablement liée à l'existence d'une phase sableuse d'abondance plus importante pour les grès des groupes Vr1 à Vr3. La forte corrélation positive entre la silice et le zirconium signifie que ces deux éléments chimiques sont liés au sein de la phase sableuse et renforce l'hypothèse d'une phase sableuse non négligeable, d'origine naturelle ou apportée par les potiers (fig. 9).

Quelques sites ont utilisé par contre des matières argileuses moins siliceuses et ayant des teneurs en potasse et rubidium plus élevées (Grès des groupes Vr4 à Vr7), le groupe Vr6 ayant les teneurs en potasse et en rubidium les plus élevées associées à des teneurs en baryum (Ba) élevées (fig. 10).

La nature argileuse de la matière première est donc différente et peut avoir contenu une fraction feldspathique potassique servant de fondant.

Le groupe Vr7 est assez différent de tous les autres et se caractérise par des teneurs nettement plus élevées en oxyde de titane, en strontium et baryum. 

les productions des fours du Lahan Saï (repérés par une étoile sur la classification) forment des sous-ensembles au sein des groupes identifiés mais quelques exemplaires de grès trouvés sur des fours autour de Ban Kruat présentent les mêmes compositions.

20 et des sites, chaque four pouvant correspondre à des potiers et à des habitudes de production et de préparation des matières premières différentes. Le facteur chronologique peut également avoir joué un rôle. Toutefois peu de groupes correspondent à un site en particulier. Nous reviendrons sur ce point.

Tableau 1. Compositions moyennes des différents groupes de grès à glaçure verte, les éléments majeurs sont donnés en pourcent d'oxyde et les traces en partie par million de métal.
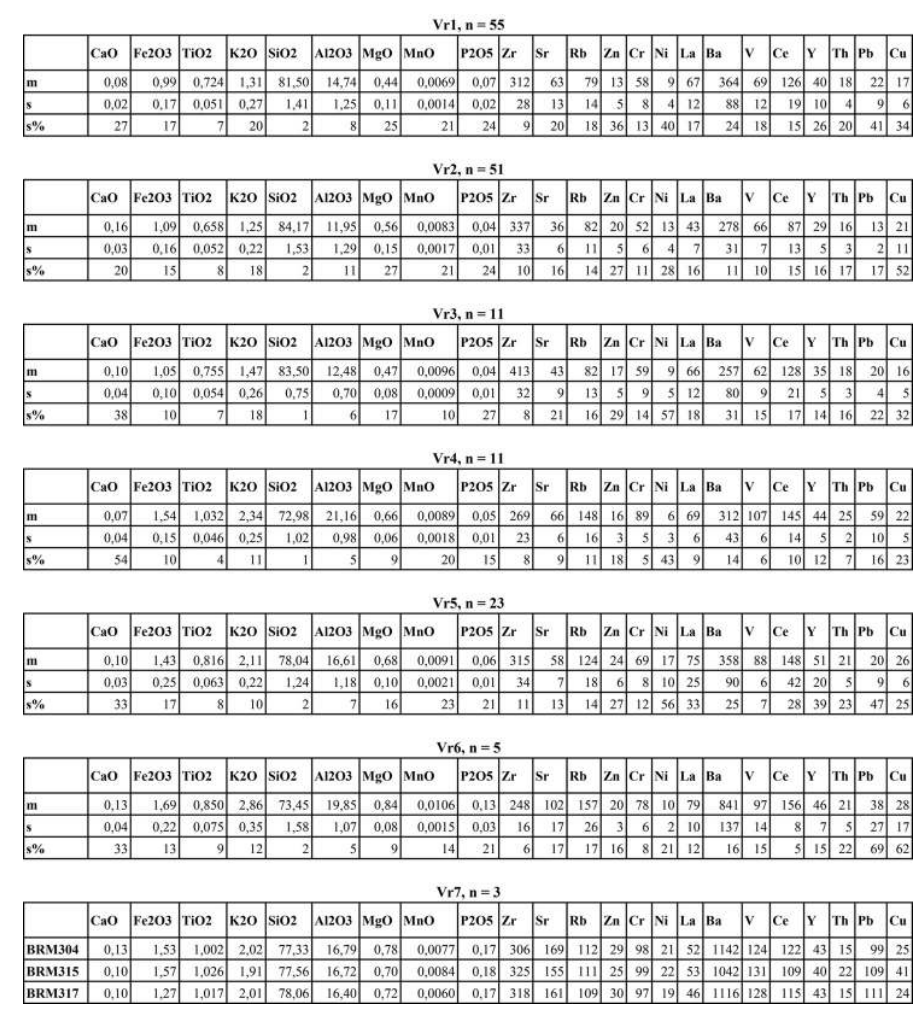
Fig. 10. Diagrammes binaires mettant en relation pour les grès à glaçure verte des groupes $\mathrm{Vr} 1$ à $\mathrm{Vr} 7$, (a) les teneurs en alumine et silice, (b) les teneurs en zirconium et silice, et (c) les teneurs en rubidium et potasse.
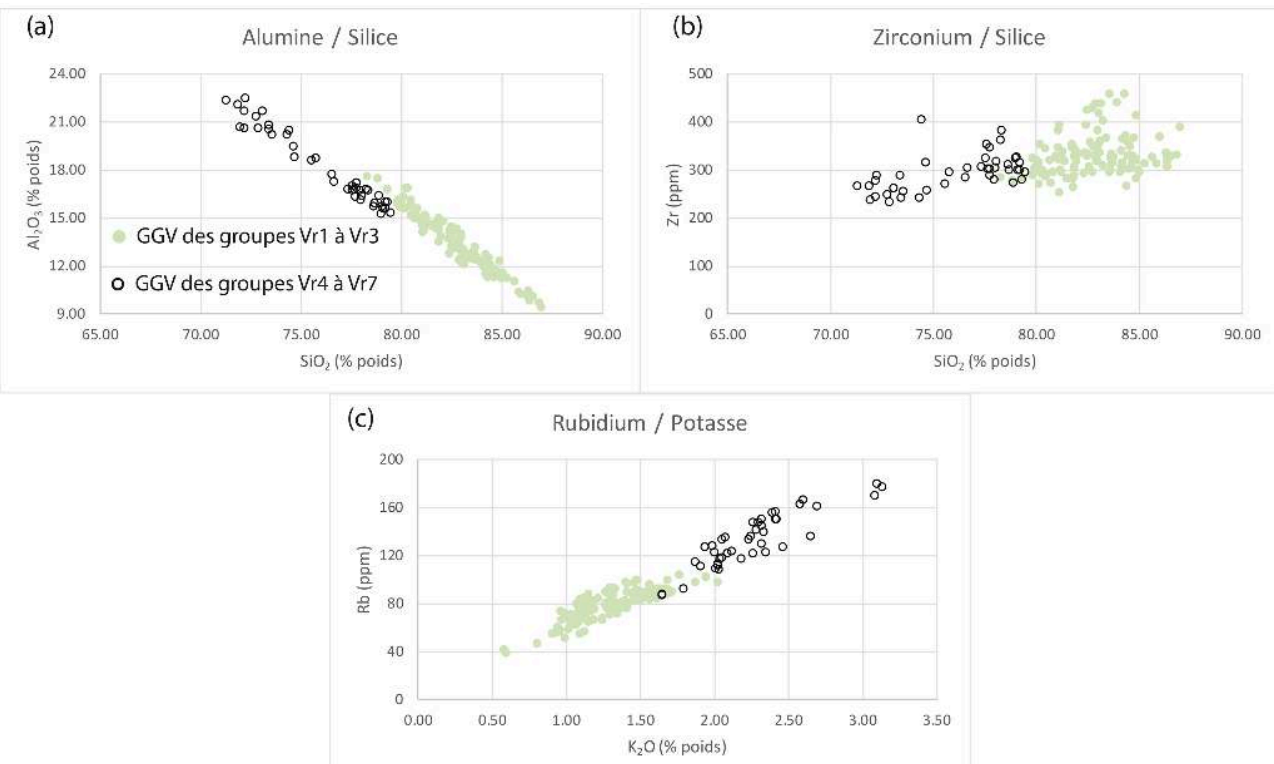

\section{Les grès à glaçure brune et grès non glaçurés}

dans le Lahan Saï et ceux produits sur les sites autour de Ban Kruat est difficile, on constate ici que les grès à glaçure brune analysés du Lahan Saï, produits principalement sur le site de Ban Rat Rak Dam, forment toujours un groupe distinct, Br6. Ce group Br6 rassemble des grès ayant des pâtes relativement claires et dont les compositions révèlent des teneurs en oxyde de fer nettement plus faibles que la plupart des autres grès à glaçure brune ou grès non glaçurés. Un autre groupe Br3b rassemble également des grès à glaçure brune ayant des teneurs en oxyde de fer faibles. Ces grès proviennent de six sites autour de Ban Kruat, et leurs compositions présentent des distinctions nettes sur plusieurs éléments chimiques indiquant l'utilisation d'un matériau argileux nettement différent. Les principaux éléments chimiques qui se démarquent sont le magnésium, manganèse, zirconium, vanadium et le cérium (cf. tableau 2).

Bulletin archéologique des Écoles françaises à l'étranger , Asie du Sud-Est continentale 
Fig. 11. Classification ascendante hiérarchique des grès à glaçure brune et grès non glaçurés retenus comme références du Buriram, réalisée sur 17 éléments chimiques, $n=180$.

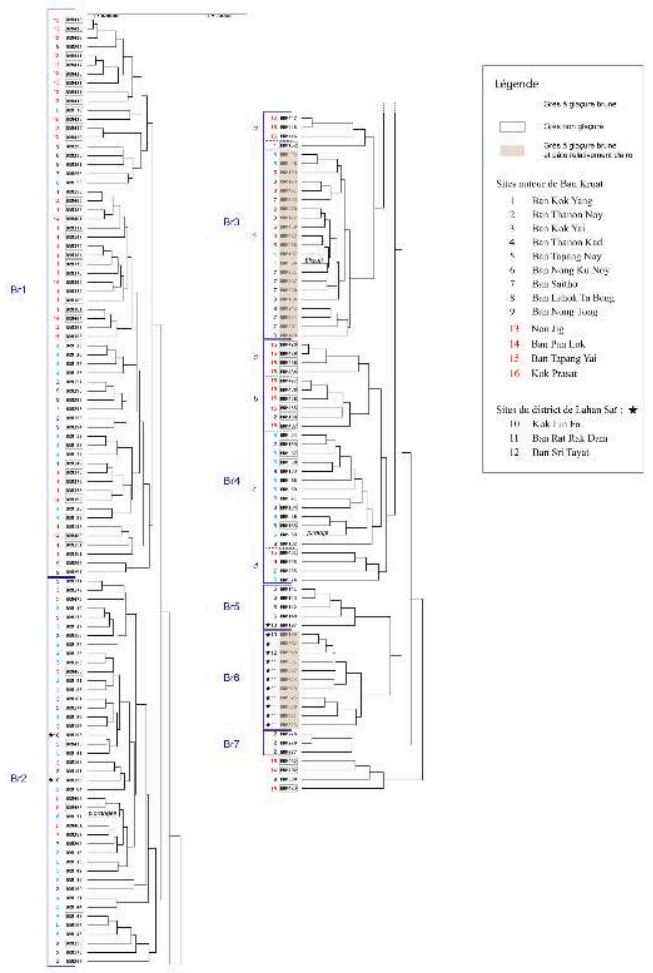

A. Desbat.

Tableau 2. Tableau des moyennes des différents groupes de composition établis. Les éléments majeurs sont donnés en pourcent d'oxyde et les traces en partie par million de métal.
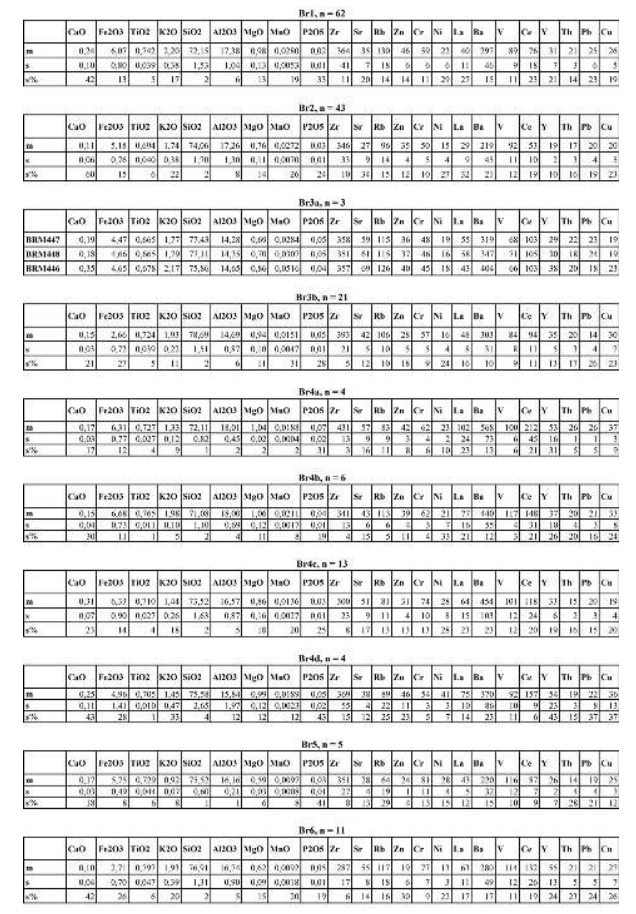

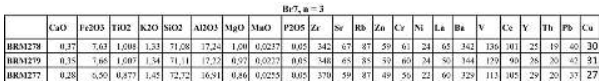




\section{Les groupes de fours en fonction des matières premières utilisées}

À partir des classifications, nous avons constaté que seuls quelques groupes de composition pouvaient correspondre à des sites particuliers. Nous avons alors cherché à mettre en relation les groupes de composition et les sites de fours.

À partir du tableau de répartition des grès dans chaque groupe de composition chimique en fonction des sites de fours dont ils proviennent, on peut voir se dessiner des zones où les potiers de différents sites de fours ont pu utiliser les mêmes matières premières (tableau 3).

Tableau 3. Tableau de répartition des grès dans chaque groupe de composition chimique en fonction des sites de fours dont ils proviennent.

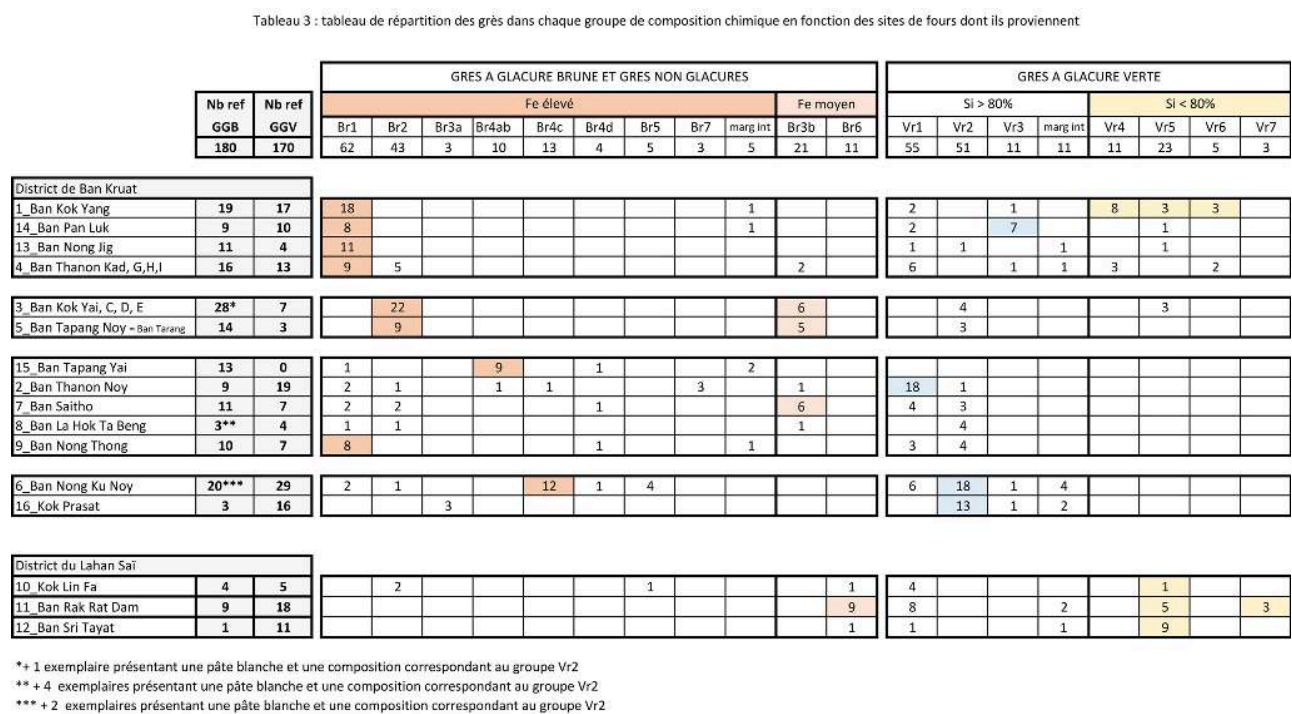

En effet, on constate que les grès à glaçure brune et les non glaçurés des sites de Ban Kok Yang, Ban Pan Luk et Ban Non Jig sont tous présents dans le même groupe de composition Br1, ce qui permet de dire que les potiers ont utilisé la même argile. Ces trois sites sont ceux situés le plus au nord/nord ouest de Ban Kruat. Le site de Ban Thanon Kad (Fours G, H, I), cette fois au nord-est, peut, en partie, être rapproché de ce groupe dans la mesure où de nombreux grès à glaçure brune ou non glaçurés se répartissent dans $\mathrm{Br} 1$. En ce qui concerne les grès à glaçure verte de ces sites, la situation n'est pas aussi claire, car ils se répartissent dans plusieurs groupes chimiques et on ne voit guère de cohérence. On peut simplement remarquer qu'à Ban Kok Yang, les potiers ont principalement utilisé des matières premières moins siliceuses.

Autre constat, les sites de Ban Kok Yai (fours C, D, E) et Ban Tapang Noy (Four J), situés également à l'est, pourraient faire partie d'un même groupe. Leurs productions sont réparties dans les mêmes groupes chimiques, $\mathrm{Br} 2$ et $\mathrm{Br} 3 \mathrm{~b}$ pour les "glaçure brune " et Vr2 pour les "glaçure verte".

On peut se demander pourquoi les sites de Ban Thanon Kad et Ban Kok Yai avaient été regroupés dans un même bloc dans la publication et la carte du Fine Art Department de 1989, car ils ne fonctionnement pas ensemble du point de vue de l'utilisation des 
matières premières. Leur grès sont répartis dans des groupes de composition bien différents.

On voit également que les productions de glaçure verte des fours de Ban Nong Ku Noy et Kok Prasat, situés au sud, peuvent correspondre aux mêmes matières premières car on ne les retrouve pratiquement que dans le groupe Vr2. Toutefois, dans ce groupe on retrouve aussi des grès d'autres sites. Cette matière première n'a pas été exclusivement utilisée par ces deux sites. Peut-être peut-on faire l'hypothèse d'un niveau de standardisation plus important à Ban Nong Ku Noy et Kok Prasat, à moins qu'il ne s'agisse d'un biais lié à notre ramassage et échantillonnage.

Par contre, pour tous les sites plus centraux sur la carte, à savoir Ban Tapang Yai, Ban Thanon Noy, Ban Saitho, Ban La Hok Ta Beng et Ban Nong Thong, il ne peut être fait de regroupement particulier en fonction des groupes de composition chimique. On peut simplement dire qu'ils ont tous utilisé des argiles très siliceuses pour produire les grès à glaçure verte. A Ban Thanon Noy, une seule argile a été utilisée car tous les grès à glaçure verte se retrouvent dans un seul groupe Vr1, alors que les grès à glaçure brune et les non glaçurés se répartissent dans 6 groupes chimiques. Le niveau de standardisation était-il plus élevé pour les grès à glaçure verte?

\section{Conclusion}

31 Les analyses chimiques ont permis de confirmer que les matières premières utilisées par les potiers du Buriram pour fabriquer les grès non glaçurés et ceux à glaçure brune étaient les mêmes, assez grossières contenant de nombreux nodules d'oxyde de fer, alors que celles utilisées pour les grès à glaçure verte sont nettement différentes, plus fines et ne contenant que très peu d'oxyde de fer. Toutefois, il est apparu que sur certains sites de fours, notamment Ban La Hok Ta Beng, Ban Saitho et Ban Kok Yai, les potiers n'ont pas sélectionné leurs argiles en fonction des objets produits, puisque l'on retrouve des grès à glaçure brune et d'autres à glaçure verte avec les mêmes compositions.

Les matières premières argileuses ayant servi à la fabrication de tous ces grès sont très riches en silice et contiennent vraisemblablement de la kaolinite qui est le minéral argileux le plus présent dans les sols du plateau au nord-ouest de la Thaïlande (Khorat plateau ${ }^{11}$. Bien souvent d'autres minéraux argileux lui sont associés, comme la vermiculite, la smectite et l'illite. La présence d'oxydes de fer sous forme d'hématite, de goethite ou de magnétite varie selon la nature du matériau parent et de la pédogénèse des sols ${ }^{12}$. Les quelques analyses chimiques d'argiles prélevées lors de nos prospections à Ban Kruat montrent des teneurs très élevées en silice et des teneurs en éléments alcalins et alcalino-terreux typiques des argiles kaolinitiques. Les teneurs en oxyde de fer varient entre 1,3 et 4,8\%. Sur les différents prélèvements d'argile effectués, un seul présentait une composition assez proche de certains grès à glaçure verte et son analyse minéralogique par diffraction des rayons $\mathrm{X}$ avait révélé la présence de kaolinite, de smectite, d'illite (peut-être interstratifiée) et de quartz.

Les analyses confirment également l'existence d'un grand nombre de groupes de composition quels que soient les types de grès produits, ces groupes compositionnels n'étant pas caractéristiques d'un site de four en particulier même si quelques rapprochements ont pu être constatés (voir supra). La variété de compositions des grès du Buriram est vraisemblablement liée à la multiplicité des fours, plus d'une centaine 
ont été signalés. Les matières premières argileuses ont pu être prélevées en de multiples petits affleurements, et à chaque site de four peut correspondre des pratiques potières différentes avec des habitudes de préparation de la pâte céramique propres. Il ne faut pas oublier le facteur chronologique qui joue certainement un rôle. On note en effet des disparités entre des sites où la production des grès verts semble dominante, comme par exemple les sites de Lahan Sai ou les fours de Ban Kok Yang et ceux où les grès à glaçure brune ou noire sont majoritaires comme Ban Kok Yai (fig. 5) et qui semblent les plus récents. Il faut peut-être également chercher une raison technique à cette évolution, comme une éventuelle raréfaction des ressources en argile blanche. C'est une hypothèse à envisager, mais difficilement démontrable, tant que ces gisements n'ont pas été identifiés.

\section{BIBLIOGRAPHIE}

BROWN et al. 1974

Roxanna Brown, Vance Childress, Michael Gluckman, «A Khmer Kiln site - Surin Province », Journal of The Siam Society 62(2), 1974, p. 239-252.

CERANGKOR 2015

Armand Desbat (dir.), avec la collaboration de Alain de Bernet, Socheat Chea, Myongduk Choi, Ranet Hong, Valérie Thirion-Merle, Kaseka Phon, Christophe Pottier, Dominique Soutif, Recherches sur les ateliers de potiers angkoriens, Rapport programme Cerangkor, MAE, 2015. Rapport inédit.

COURBIN 1988

Paul Courbin, La fouille du Sras Srang, Paris, EFEO, Collection de textes et documents sur l'Indochine XVII, 1988.

DESBAT 2020

Armand Desbat, « New data on the chronology of Khmer stonewares », in Helen Lewis (éd.), Papers from the Fourteenth International Conference of the European Association of Southeast Asian Archaeologists EurASEAA14, Volume II, Material Culture and Heritage, Oxford, Archeopress, 2020, p. 63-75.

EA 2009

Darith Ea, « New Data on the Distribution of Khmer ceramic Kilns and the Study of Ceramics », in B. McCarthy, E.S. Chase, L.A. Cort, J.G. Douglas, P. Jett (éd.), Scientific Research on Historic Asian Ceramics: Proceeding of the fourth Forbes Symposium at the Freer Gallery of Art and Arthur M. Sackler Gallery, Washington DC, Archetype Publications with the Freer Gallery of Art, 2009, p. 207-214.

EA, 2010

Darith Ea, Angkorian Stoneware Ceramics : The Evolution of Kiln Structure and Ceramic Typology, Dissertation submitted for the degree of Doctor of Philosophy, Osaka Othani University, 2010.

FINE ART DEPARTMENT (Thailand) 1989

Fine Art Department, Ancient Kiln Sites in Buriram Province, Bangkok, Fine Arts Department, 1989. 
GROSLIER 1957

Bernard Philippe Groslier, « Excavations at the Royal Palace of Angkor Thom, preliminary report ", in Dénes Sinor (éd.), Proceedings of the Twenty-Third International Congress of Orientalists, Cambridge, 21 $1^{\text {st }}-28^{\text {th }}$ August 1954, Londres, The Royal Asiatic Society, p. 28.

GROSLIER, 1964

Bernard Philippe Groslier, « Rapport de la conservation d'Angkor (RCA), Banteay Kdei - Sras Srang, esplanade occidentale », Archives de l'École française d'Extrême Orient, Paris, 1964.

Khwanyuen 1985

Sathaporn Khwanyuen, « The Excavations of Baranse Kiln Site, Thailand », SPAFA Final Report, Technical Workshop on Ceramics, Bangkok, SPAFA, 1985, p. 137-168.

MAFKATA-CERANGKOR 2016

Christophe Pottier, Armand Desbat (dir.), avec la collaboration de Alain de Bernet, Myongduk Choi, Ranet Hong, Valérie Thirion-Merle, Nicolas Nauleau, S. San, Dominique Soutif, Sary Van, Rapport programme MAFKATA-CERANGKOR, MAE, 2016. Rapport non publié.

MAFKATA-CERANGKOR 2018

Christophe Pottier, Armand Desbat (dir.), avec la collaboration de Socheat Chea, Valérie ThirionMerle, Gisela Thierrin-Michael, Nicolas Nauleau, Sophom Nhoem, Rapport programme MAFKATACERANGKOR, MAE, 2017. Rapport non publié.

NATTHAPATRA 1990

Chandavij N. Natthapatra, « Ancient Kiln sites in Buriram Province, Northeastern Thailand », in Ho Chuimei (éd.), Ancient Ceramic Kiln Technology in Asia, Hong Kong, University of Hong Kong, Centre of Asian Studies, 1990, p. 230-243.

ROONEY, SMITHIES 1992

Dawn F. Rooney, Michael Smithies, « The Khmer kilns of ban Ya Kha », Journal of Siam Society, 85(1/2), p. 151-159.

SRISUCHAT, SRISUCHAT 1989

Tharapong Srisuchat, Amara Srisuchat, « Introducing Buriram Ceramics and Kilns », The Silpakorn Journal 33(2), p. 42-52.

SUDDHIPRAKARN et al. 1985

Anchalee Suddhiprakarn, Irb Kheoruenromne, Pramualpong Sindhusen, Kannica Yoothong,

«Clay minerals and iron oxides of selected red and yellow soils in north-east plateau and southeast coast, Thailand », Kasetsart Journal. Natural Science 19, 1985, p. 265-271.

THIRION-MERLE 2014

Valérie Thirion-Merle, «Spectrométrie de fluorescence X », in P. Dillmann, L. Bellot-Gurlet (dir.), Circulation et provenance des matériaux dans les sociétés anciennes, Paris, Ed. des Archives contemporaines, Collection « Sciences Archéologiques », 2014, p. 291-297.

THIRION-MERLE et al. 2019

Valérie Thirion-Merle, Gisela Thierrin-Michael, Armand Desbat, « Archaeometrical study of Khmer Stoneware of the Angkorian period: some results of the Cerangkor Project ", Archaeometry 61(6), 2019, p. 1246-1263.

THIRION-MERLE et al. 2020

Valérie Thirion-Merle, Gisela Thierrin-Michael, Armand Desbat, Ranet Hong, « The kiln sites of the "Torp Chey" area (Cambodia): production and distribution », BEFEO 106, p. 195-223. 
WAKSMAN 2014

Sylvie Yona Waksman, «Études de provenance de céramiques ", in Philippe Dillmann, Ludovic Bellot-Gurlet (dir.), Circulation et provenance des matériaux dans les sociétés anciennes, Paris, Ed. des Archives contemporaines, Collection « Sciences Archéologiques », 2014, p. 195-215.

YOOTHONG et al. 1997

Kannica Yoothong, Lek Moncharoen, Pisuth Vijarnson, Hari Eswaran, « Clay mineralogy of Thai soils », Applied Clay Science 11, 1997, p. 357-371.

\section{NOTES}

1. GROSLIER 1957, 1964 ; COURBIN 1988 ; EA 2009, 2010.

2. Initié en 2008, CERANGKOR est un programme de recherche collaboratif du CNRS, en association avec l'École française d'Extrême-Orient (Centre de Siem Reap, Cambodge) et l'APSARA (Authority for the Protection and Management of Angkor and the Region of Siem Reap). Il a été financé par la commission des fouilles du Ministère des Affaires étrangères et européennes.

3. DESBAT 2020.

4. THIRION-MERLE et al. 2019 ; THIRION-MERLE et al. 2020.

5. KHWANYUEN 1985 ; SRISUCHAT, SRISUCHAT 1989 ; NATTHAPATRA 1990.

6. BROWN et al. 1974 ; ROONEY, SMITHIES 1992 ; CERANGKOR 2015 ; MAFKATA-CERANGKOR 2016, 2018.

7. THIRION-MERLE 2014 ; WAKSMAN 2014.

8. Mafkata-Cerangkor 2018.

9. La méthode mise en œuvre est une classification ascendante hiérarchique utilisant les données chimiques centrées réduites, les distances euclidiennes et le critère d'agrégation dit du lien moyen. Les calculs ont été réalisés à partir de 17 constituants sur les 24 analysés.

10. Ne sont pas pris en considération dans cette classification les grès à glaçure brune ayant été fabriqués avec les mêmes argiles que les grès à glaçure verte.

11. SUDDHIPRAKARN et al. 1985.

12. YOOTHONG et al. 1997. 
INDEX

Année de l'opération : 2019

peuples https://ark.frantiq.fr/ark:/26678/pcrtkmkmzSc1dJ

chronologie https://ark.frantiq.fr/ark:/26678/crtLHPYnHVML5

lieux https://ark.frantiq.fr/ark:/26678/pcrtOUVodanE3h

Thèmes : EFEO

sujets https://ark.frantiq.fr/ark:/26678/pcrt1DMOWvDF4j, https://ark.frantiq.fr/ark:/26678/ pcrtt7G9pnQlu1, https://ark.frantiq.fr/ark:/26678/pcrtxP5I53Pj0s

\section{AUTEURS}

\section{VALÉRIE THIRION-MERLE}

CNRS, UMR 5138 Archéologie et Archéométrie ArAr, Maison de l’Orient et de la Méditerranée Jean Pouilloux

\section{ARMAND DESBAT}

CNRS, UMR 5138 Archéologie et Archéométrie ArAr, Maison de l'Orient et de la Méditerranée Jean Pouilloux

\section{RANET HONG}

EFEO Centre de Siem Reap, Cambodge 